\title{
HYPERBOLIC STRUCTURES FOR SURFACES OF INFINITE TYPE
}

\author{
ARA BASMAJIAN
}

\begin{abstract}
Our main objective is to understand the geometry of hyperbolic structures on surfaces of infinite type. In particular, we investigate the properties of surfaces called flute spaces which are constructed from infinite sequences of "pairs of pants," each glued to the next along a common boundary geodesic. Necessary and sufficient conditions are supplied for a flute space to be constructed using only "tight pants," along with sufficient conditions on when the hyperbolic structure is complete. An infinite version of the Klein-Maskit combination theorem is derived.

Finally, using the above constructions a number of applications to the deformation theory of infinite type hyperbolic surfaces are examined.
\end{abstract}

\section{INTRODUCTION}

A fuchsian group is said to be a pair of pants if its associated quotient surface is topologically a sphere with three holes. The pair of pants is tight if one of the holes is conformally a puncture. We say that two boundary elements that generate a pair of (tight) pants are standard generators if each one represents a different boundary component and their product represents the (puncture) third boundary component.

We consider hyperbolic surfaces of infinite type, that is, surfaces with an infinitely generated fundamental group. In particular, we construct surfaces called (tight) flute spaces from a union of pairs of (tight) pants each glued to the next along a common boundary geodesic (see Figure 1). These common boundary geodesics on the surface form what we call a nested sequence of geodesics. Let $d_{i}$ be the hyperbolic distance between the $i$ th and $(i+1)$ st geodesics. We show (Theorem 1) that given any sequence of positive numbers $\left\{d_{i}\right\}$ there exists a hyperbolic structure for a flute space having a nested sequence of geodesics of hyperbolic distances $\left\{d_{i}\right\}$. In particular, if the series $\sum d_{i}$ converges and if there is not too much twisting in the nest (see $\S 3$ for the definition of the twist parameters), then the lifts of the nested sequence to the universal cover form a nested sequence of axes of hyperbolic elements which converge to a geodesic. This phenomenon has gone unnoticed in the literature. Next we supply necessary and sufficient conditions (Theorem 2), in the form

Received by the editors July 27, 1990 and, in revised form, December 18, 1990.

1980 Mathematics Subject Classification (1985 Revision). Primary 30F35; Secondary 20H10, $32 \mathrm{G} 15$.

Key words and phrases. Fuchsian group, hyperbolic surface, Riemann surface, pair of pants. 
of inequalities involving distances between geodesics, for the existence of a hyperbolic structure on a tight flute. In addition, we show (Theorem 4) that the fuchsian group associated to a tight flute is of the first kind if $\sum d_{i}=\infty$.

One of the central results in the theory of fuchsian groups (the Nielsen isomorphism theorem) is that every type preserving isomorphism (that is, one that takes boundary geodesics to boundary geodesics and parabolics to parabolics) between finitely generated torsion-free fuchsian groups is a topological deformation (that is, it is induced by a topological map of $\overline{\mathbb{H}}^{2}$ ). We show that for infinitely generated groups this theorem does not hold. More precisely, we identify why the proof from the finitely generated case does not extend to the general case. See the examples in $\S 9$.

Another of the main tools used in the study of fuchsian groups is the KleinMaskit combination theorem [M] which prescribes conditions for when one can glue fundamental polygons to construct new fundamental polygons (and in turn construct new surfaces out of simpler ones). We supply sufficient conditions (Theorem 5) on an infinite number of polygons, so that when glued together they form a new fundamental polygon.

Theorems 4 and 5 can be thought of as prescribing conditions for when the infinite process of hyperbolic gluing yields a complete hyperbolic surface. More precisely, we start with a sequence of hyperbolic surfaces with geodesic boundary and (hyperbolically) glue each one to the next along common boundary geodesics. This process produces an end of the so-called infinite type. The hyperbolic structure for this end is complete, if the series of lengths between each of the geodesics we glued along is divergent.

An enormous amount of work has been done in the study of Riemann surfaces of infinite type (in particular, for the study of plane domains with infinite connectivity), as exemplified in the books of Ahlfors-Sario [A-S] and NakaiSario [N-S], dealing with classification theory. The classification theoretic approach to studying plane domains (and more generally open Riemann surfaces) is to try and understand the functions the domains carry. For example, the complex plane does not carry a harmonic function with finite Dirichlet integral but the unit disc does. This literature is rich with beautiful theorems and constructions using complex function theory.

In another direction infinite-dimensional Teichmüller theory has seen much development through the works of Bers, Earle, Gardiner, Kra, Maskit, Royden, and many others (see Gardiner's Teichmüller theory [G] for an extensive bibliography). These results are for the most part of an analytical nature.

Both of the approaches mentioned above use little or no hyperbolic geometry. On the other hand, there is an extensive hyperbolic geometric theory of finite type Riemann surfaces. Starting with the well-known geometric coordinates for Teichmüller space, the Fenchel-Nielsen coordinates [A], and moving all the way into the Thurston theory of geodesic laminations [T], hyperbolic geometry has played a profound part in shaping the theory of finite type Riemann surfaces.

This paper is an attempt to bring hyperbolic geometry to center stage for the study of infinite type Riemann surfaces. The starting point of this investigation is the observation that every infinite type Riemann surface contains a nested sequence of closed geodesics. Our aim is to analyze these nests and to determine to what extend their behavior is related to the geometry of the surface. The 
main class of examples we look at, flute spaces, already exhibit much of the complexity and diversity inherent in surfaces of infinite type.

Other writers who have investigated infinitely generated fuchsian groups include Keen [K] and Purzitsky [P-1, P-2].

Most of the contents of this paper are contained in the doctoral dissertation [B-1]. The author wishes to thank his advisor Bernard Maskit for his encouragement and shared wisdom during the preparation of this manuscript.

\section{Statement of Results}

We say that $G$ is a hyperbolic structure for $S$ if $G$ is a fuchsian group and $\mathbb{H}^{2} / G$ is topologically equivalent to $S . S$ together with its hyperbolic structure $G$ is said to be a hyperbolic surface. The Nielsen convex region of $G$ is denoted $N(G)$.

A hyperbolic surface $S$ is a flute space if its associated hyperbolic structure $G$ is of the form $\left\langle G_{i}\right\rangle_{i=1}^{\infty}$, where the $G_{i}$ are pairs of pants and for each $i$,

$$
N\left(G_{i}\right) \cap N\left(G_{i+1}\right)=A\left(g_{i+1}\right) ;
$$

here $g_{i+1}$ is a primitive boundary hyperbolic element in both $G_{i}$ and $G_{i+1}$. $S$ is said to be a tight flute if in addition $G_{1}$ is a pair of pants with two punctures and a hole and the $G_{i}$, for $i=2,3, \ldots$, are tight pants.

We observe that the $G_{i}$ give a pants decomposition $\left\{P_{i}\right\}$ of $S$ and the pants decomposition naturally gives us a nested sequence of simple closed curves on $S$ denoted $\left\{w_{i}\right\}$. (See Figure 1 on the next page.)

A standard set of generators $\left\{g_{i}\right\}$ for $G$ relative to the decomposition $\left\{P_{i}\right\}$ is a set of generators for $G$ satisfying the following conditions:

(i) For each $i$, the projection of the axis of $g_{i}$ to $S$ is freely homotopic to $w_{i}$. (We allow the possibility that $g_{1}$ is a parabolic in which case we project the horocycle for $g_{1}$, called the axis of $g_{1}$, whose image in $S$ bounds a punctured disc of area one. For instance, $g_{1}$ will be parabolic if $G$ is a tight flute.)

(ii) Each pair $\left(g_{i}, g_{i+1}\right)$ forms standard generators for the pair of pants $P_{i}$. Hyperbolic standard generators for a pair of pants were defined in [B-2]; we state the definition here in a more general form. We say that two nonelliptic elements $\gamma$ and $\beta$ form standard generators for a pair of pants $G$ if

(a) $\gamma$ and $\beta$ are each primitive boundary elements in $G$ that represent two of the boundary components on $\mathbb{H}^{2} / G$ with $\langle\gamma, \beta\rangle=G$, and

(b) after possibly changing the orientations of $\gamma$ and $\beta$ so that their axes lie to the right of each other, the product $\beta \gamma$ is a primitive boundary element of $G$ which represents the third boundary component (or equivalently, since $\beta \gamma$ is conjugate to $\gamma \beta$ in $G, \gamma \beta$ is a primitive boundary element which represents the third boundary component). Here, boundary element means either a parabolic or boundary hyperbolic element. More details can be found in $\S 5$.

Throughout this section $S$ is a flute space with $\left\{P_{i}\right\}$ a fixed pants decomposition of $S$.

The axes of a standard set of generators form a nested sequence of geodesics in $\mathbb{H}^{2}$ having coordinates $\left(\left(d_{i}\right),\left(s_{i}\right)\right)$. Here $d_{i}$ measures the hyperbolic distance from the axis of $g_{i}$ to the axis of $g_{i+1}$, and $s_{i}$ measures the amount of twisting along the axis of $g_{i+1}$. (See $\S 3$ for precise definitions.) We can now state our main results. 
424

ARA BASMAJIAN

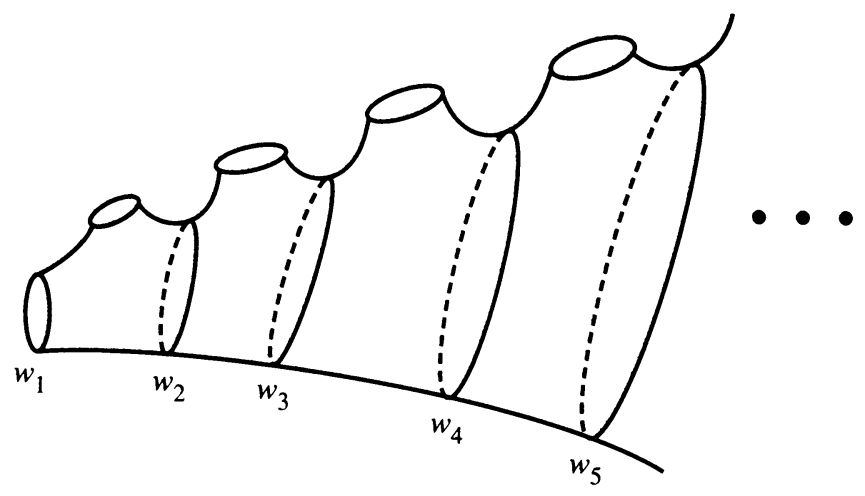

flute space

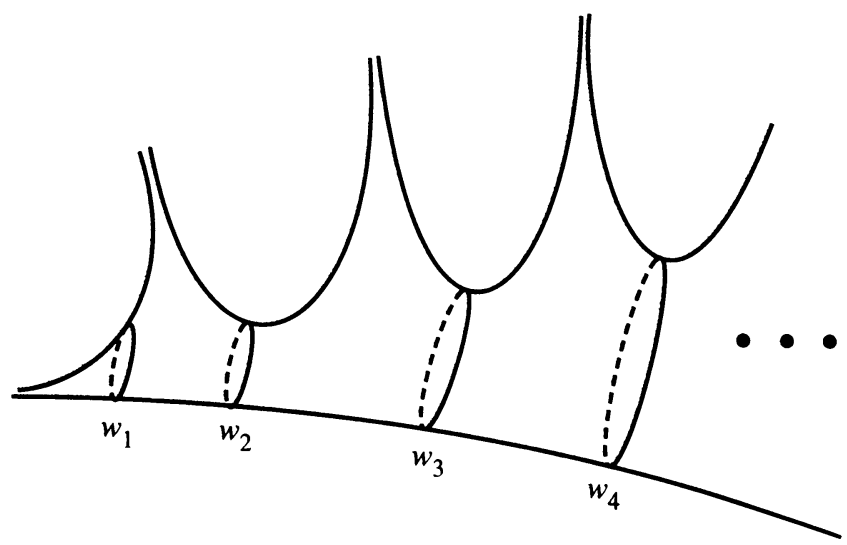

tight flute

FIGURE 1

Theorem 1. Given any set of positive real numbers $\left\{d_{i}\right\}$ and real numbers $\left\{s_{i}\right\}$, there exists a flute space having a standard set of generators with coordinates $\left(\left(d_{i}\right),\left(s_{i}\right)\right)$.

Equivalently, this shows that any nested sequence of geodesics in $\mathbb{H}^{2}$ can be realized as the nest of axes for a standard set of generators of a fuchsian group whose associated quotient surface is a flute space.

Next we look at what can happen if the flute is required to be tight.

Theorem 2. Let $\left\{d_{i}\right\}$ be positive real numbers and let $\left\{s_{i}\right\}$ be a set of real numbers. Then $\left(\left(d_{i}\right),\left(s_{i}\right)\right)$ are coordinates for a standard set of generators $\left\{g_{i}\right\}$ for a tight flute if and only if

(*) $\quad t_{n}>\log 2$ for all $n$ odd and $t_{n}<\log 2$ for all $n$ even, where $t_{n}=\sum_{i=1}^{n}(-1)^{i+1} d_{i}$.

Observe that condition $(*)$ does not involve the twist coordinates $\left(s_{i}\right)$. Hence any amount of twisting is possible for a tight flute. There are many sequences 
that satisfy condition $(*)$ and many that do not (see the corollary to Theorem 2 in $\S 8)$.

One can think of Theorems 1 and 2 as geometric realization theorems.

The construction of flute spaces involves understanding how to piece together hyperbolic structures for infinitely many pairs of pants. The following theorem, which we call the fundamental theorem, guarantees that under certain geometric conditions the limit of fuchsian groups is a fuchsian group.

Theorem 3. Let $\left\{G_{n}\right\}_{n=1}^{\infty}$ be fuchsian groups with $\left\langle g_{n}\right\rangle$ and $\left\langle g_{n+1}\right\rangle$ nonconjugate primitive boundary hyperbolic subgroups in $G_{n}$ so that, for each $n$,

$$
N\left(G_{n}\right) \cap N\left(G_{n+1}\right)=A\left(g_{n+1}\right) .
$$

Let $G=\left\langle G_{n}\right\rangle_{n=1}^{\infty}$. Then

(I) $G$ is a fuchsian group (that is, a hyperbolic structure).

(II) $N(G) / G$ is topologically the union of surfaces $\left\{N\left(G_{n}\right) / G_{n}\right\}$ each glued to the next along the simple closed dividing geodesics $\left\{\pi A\left(g_{i}\right)\right\} \quad(\pi$ is the covering map to the quotient $\left.\mathbb{H}^{2} / G\right)$. Furthermore, $N\left(G_{n}\right) / G_{n}$ isometrically embeds in $N(G) / G$.

One purpose of using the coordinates $\left(\left(d_{i}\right),\left(s_{i}\right)\right)$ for standard generators is that they allow us to have some control of the geometry at "infinity" for the fuchsian group representing the flute space. Specifically, we prove the following theorem.

Theorem 4. Suppose $\left\{g_{i}\right\}$ is a set of standard generators with coordinates $\left(\left(d_{i}\right),\left(s_{i}\right)\right)$ for a tight flute $G$. Then $G$ is of the first kind if the series $\sum d_{i}$ diverges. If the series $\sum d_{i}$ and $\sum\left|s_{i}\right|$ converge, then $G$ is of the second kind.

Suppose we have groups $\left\{G_{n}\right\}$ and hyperbolic elements $g_{n}$ and $g_{n+1}$ satisfying the hypotheses in Theorem 3 . Define $G^{n}$ to be $\left\langle G_{1}, \ldots, G_{n}\right\rangle$, and for each $i$ let $D_{i}$ be a fundamental polygon for $G_{i}$. Let $B\left(g_{n}\right)$ be the component (half-space) of $\mathbb{H}^{2}-A\left(g_{n}\right)$ containing the Nielsen region $N\left(G_{n-1}\right)$. Denote the other component by $B^{\prime}\left(g_{n}\right)$. We say that the $\left\{D_{i}\right\}$ pairwise satisfy the compatibility conditions in the combination theorem if for each $i, D_{i}$ and $D_{i+1}$ satisfy the hypotheses in the combination theorem; that is,

(a) $D_{i} \cap B\left(g_{i+1}\right) \subset D_{i+1} \cap B\left(g_{i+1}\right)$,

(b) $D_{i+1} \cap B^{\prime}\left(g_{i+1}\right) \subset D_{i} \cap B^{\prime}\left(g_{i+1}\right)$, and

(c) the sides of $D_{i}$ that are paired by $g_{i+1}$ lie on the same geodesic lines as the sides of $D_{i+1}$ that are paired by $g_{i+1}$.

The following theorem can be thought of as an infinite version of the KleinMaskit combination theorem.

Theorem 5. Let $\left\{G_{n}\right\}_{n=1}^{\infty}$ be fuchsian groups with $\left\langle g_{n}\right\rangle$ and $\left\langle g_{n+1}\right\rangle$ nonconjugate primitive boundary hyperbolic subgroups in $G_{n}$ so that, for each $n$ :

(I) $N\left(G_{n}\right) \cap N\left(G_{n+1}\right)=A\left(g_{n+1}\right)$.

(II) The distance between the closed geodesics associated to $g_{n}$ and $g_{n+1}$ on the quotient surface $N\left(G_{n}\right) / G_{n}$ is equal to $\rho\left(g_{n}, g_{n+1}\right)$. Furthermore, let $\left\{D_{n}\right\}$ be fundamental polygons for $\left\{G_{n}\right\}$ which pairwise satisfy the compatibility conditions in the combination theorem. Set $G=\left\langle G_{n}\right\rangle_{n=1}^{\infty}$.

Then the intersection $\cap D_{n}$ is a fundamental polygon for $G$ if

$$
\sum \rho\left(g_{n}, g_{n+1}\right)=\infty
$$


where we use the notation $\rho\left(g_{n}, g_{n+1}\right)$ to stand for the hyperbolic distance from the axis of $g_{n}$ to the axis of $g_{n+1}$.

Theorem 5 no longer holds if the series $\sum \rho\left(g_{n}, g_{n+1}\right)$ converges (see $\S 9$ for an example). Condition (II) is automatically satisfied if $G$ is a flute space with standard generators $\left\{g_{n}\right\}$.

The contents of this paper are made up of nine sections. Section 2 will set up some notation and discuss some definitions and known theorems which will be needed later in the paper.

In $\S 3$, we develop a theory of nested sequences of geodesics. Although the development is at an elementary level, it is crucial for our constructions. Sections 4-7 contain the proofs of Theorems 1-5.

Section 8 contains a corollary to Theorem 2 and a proposition which is a consequence of some of the work in $\S 5$. The corollary supplies us with a number of examples of sequences $\left\{d_{i}\right\}$ that satisfy condition $(*)$. The proposition is concerned with determining the lengths of closed geodesics in a nested sequence for a flute space when given the coordinates for a standard set of generators on the flute space.

Finally, in $\S 9$ we list a number of examples. Our first example illustrates the fact that Theorem 5 does not hold if the series $\sum \rho\left(g_{n}, g_{n+1}\right)$ converges.

One might think that the result in Theorem 4-the tight flute is of the first kind if the series $\sum d_{i}$ diverges-can be improved by simply requiring that the nest of axes for the standard generators go to the boundary of the hyperbolic plane. The next example shows that this requirement is not enough to guarantee that the tight flute is of the first kind.

Our last example in $\S 9$ is of a type preserving isomorphism that is not a topological deformation. This shows that the statement of the Nielsen isomorphism theorem does not extend to infinitely generated groups.

\section{Preliminaries AND NOtation}

This section will serve as a very brief introduction to fuchsian groups; the main objective is to set up notation for the sections to follow. For a more detailed account on the theory of fuchsian groups and Riemann surfaces the reader is referred to the books of Maskit [M] and Beardon [Be].

Two models of the hyperbolic plane $\mathbb{H}^{2}$ are the upper half-plane $\{z \in \mathbb{C}$ : $\operatorname{Im}(z)>0\}$ and the Poincaré disc $\{z \in \mathbb{C}:|z|<1\}$. Their line elements are $d s=\frac{|d z|}{(\operatorname{Im}(z))}$ and $d s=\frac{2|d z|}{1-|z|^{2}}$, respectively. Both of these models have Euclidean boundaries which can be identified with $\partial \mathbb{H}^{2}$, the boundary of $\mathbb{H}^{2}$. Thus the hyperbolic boundary of the upper half-plane is the extended real numbers $\mathbb{R} \cup \infty$ and for the Poincare disc it is the unit circle. We let $\rho(\cdot, \cdot)$ be the hyperbolic distance between two points of $\mathbb{H}^{2}$.

The geodesics for the upper half-plane are vertical lines and arcs of circles which are orthogonal to the real line. For the Poincare disc, the geodesics are straight lines through the origin and arcs of circles which are orthogonal to the unit circle. We denote the geodesic with endpoints $a$ and $b$ on the boundary of $\mathbb{H}^{2}$ by $[a, b]$.

The group of orientation-preserving isometries of the upper half-plane (with 
the hyperbolic metric) is made up of the Möbius transformations

$$
z \mapsto \frac{a z+b}{c z+d}
$$

where $a d-b c=1$ and $a, b, c, d \in \mathbb{R}$. This group can be naturally identified with the projectivized special linear group, $\operatorname{PSL}(2, \mathbb{R})$.

A hyperbolic element $h$ has a unique invariant geodesic line in $\mathbb{H}^{2}$, called the axis of $h$ and denoted $A(h)$. If $z$ is a point on $A(h)$, the translation length of $h$, denoted $T(h)$, is the distance $\rho(z, h(z))$.

A fuchsian group is a discrete subgroup of orientation preserving isometries of $\mathbb{H}^{2}$. We say that the fuchsian group $G$ is a hyperbolic structure for the surface $S$ if $\mathbb{H}^{2} / G$ is topologically equivalent to $S$. The action of a fuchsian group $G$ on the closure of the hyperbolic plane $\overline{\mathbb{H}}^{2}$ naturally breaks up into the set of discontinuity $\Omega(G)$ and the limit set $\Lambda(G)$. The limit set of $G$ lies entirely in $\partial \mathbb{H}^{2}$.

If $\Lambda(G)=\partial \mathbb{H}^{2}$, then we say that $G$ is a fuchsian group of the first kind. Otherwise, $\Omega(G) \cap \partial \mathbb{H}^{2}$ is the union of open intervals and $G$ is said to be of the second kind. These intervals are known as intervals of discontinuity. An open half-space $B \subset \mathbb{H}^{2}$ which "bounds" an interval of discontinuity is called a boundary half-space for $G$. If there exists a hyperbolic element $g \in G$ whose axis "bounds" this interval of discontinuity, we say that $g$ is a boundary hyperbolic element with boundary half-space $B$. The cyclic subgroup generated by $g,\langle g\rangle$, is referred to as a boundary hyperbolic subgroup. A boundary element for $G$ is either a boundary hyperbolic or a parabolic element.

Two subgroups $H_{1}$ and $H_{2}$ of $G$ are said to be $G$-conjugate if there exists an element $g \in G$ so that $g H_{1} g^{-1}=H_{2}$.

We say that a subset $X$ of $\overline{\mathbb{H}}^{2}$ is precisely invariant under the subgroup $\Gamma$ of $G$ if

(i) $\gamma X=X$ for all $\gamma \in \Gamma$ and

(ii) $g X \cap X=\varnothing$ for all $g \in G-\Gamma$.

If $X$ is open this says exactly that $X / \Gamma$ isometrically imbeds into $\mathbb{H}^{2} / G$.

Suppose $G$ is a torsion-free fuchsian group. Then $\mathbb{H}^{2} / G$ is an oriented hyperbolic surface of constant negative curvature whose fundamental group is isomorphic to $G$. The natural projection map $\pi: \mathbb{H}^{2} \rightarrow \mathbb{H}^{2} / G$ is a covering with group of deck transformations $G$.

A fuchsian group $G$ is of type $(g ; n ; m)$ if $\mathbb{H}^{2} / G$ is a compact surface of genus $g$ with $n$ points and $m$ conformal discs removed. If $g=0$ and $m+n=3$, we say that $\mathbb{H}^{2} / G$ (and likewise $G$ ) is a pair of pants.

The Nielsen (convex) region $N(G)$ for a fuchsian group $G$ is the smallest nonempty $G$-invariant closed convex subset of $\mathbb{H}^{2}$. Equivalently, $N(G)$ is $\mathbb{H}^{2}-\{$ boundary half-spaces of $G\}$.

One of the essential ways of understanding the action of a fuchsian group on $\mathbb{H}^{2}$ is by way of fundamental polygons.

A (convex) polygon $D$ in $\mathbb{H}^{2}$ is the intersection of countably many open half-spaces, with the property that any compact set in $\mathbb{H}^{2}$ intersects only finitely many geodesics which define the half-spaces. The geodesic segments that make up the boundary of $D$ are called sides. Two sides meet at a vertex. 
Definition. Let $G$ be a fuchsian group. A polygon $D$ is a (convex) fundamental polygon for $G$ if the following conditions hold:

(i) $g D \cap D=\varnothing$ for all nontrivial $g \in G$.

(ii) $\bigcup_{g \in G} g \bar{D}=\mathbb{H}^{2}$.

(iii) The sides of $D$ are paired by elements of $G$.

(iv) (local finiteness) Any compact set intersects only finitely many translates of $D$.

An important feature of a fundamental polygon lies in the fact that $\bar{D} / G$ is hyperbolically isometric to $\mathbb{H}^{2} / G$.

At this point there arises a natural question. Which polygons with sides paired by (orientation-preserving) isometries of $\mathbb{H}^{2}$ are fundamental polygons for fuchsian groups? Poincaré's theorem supplies us with the answer. We content ourselves with a restricted form of the theorem. The general form can be found in the book [M].

Poincaré's Polygon Theorem. Suppose $D$ is a finite sided (convex) polygon in $\mathbb{H}^{2}$, satisfying the following conditions.

(i) the sides of $D$ are paired by isometries of $\mathbb{H}^{2}$;

(ii) $\gamma D \cap D=\varnothing$ for all the side pairing transformations $\Gamma$;

(iii) the orbit of a vertex under the side pairing transformations is the set of all vertices of $D$;

(iv) the sum of the angles at the vertices is equal to $2 \pi$; and

(v) $D$ has no sides that meet tangentially on the boundary of $\mathbb{H}^{2}$.

Then $D$ is a fundamental polygon for the fuchsian group generated by the side pairing transformations.

A basic tool in the theory of fuchsian groups is the combination theorem. It enables one to construct complicated fuchsian groups out of simple ones. Once again we content ourselves with a special case.

Suppose $h$ is a boundary hyperbolic in a fuchsian group $G$. Denote by $B(h)$ the component of $\mathbb{H}^{2}-A(h)$ which contains $N(G)$. Let $B^{\prime}(h)$ be the other component.

The Klein-Maskit Combination Theorem. Let $D_{1}$ and $D_{2}$ be (convex) fundamental polygons for the fuchsian groups $G_{1}$ and $G_{2}$, respectively. Suppose $h$ is a primitive boundary hyperbolic in both $G_{1}$ and $G_{2}$ satisfying the following conditions:

(i) $N\left(G_{1}\right) \cap N\left(G_{2}\right)=A(h)$;

(ii) $D_{1} \cap B(h) \subset D_{2} \cap B(h)$;

(iii) $D_{2} \cap B^{\prime}(h) \subset D_{1} \cap B^{\prime}(h)$; and

(iv) the sides of $D_{1}$ that are paired by $h$ lie on the same geodesic lines as the sides of $D_{2}$ that are paired by $h$.

Then $\left\langle G_{1}, G_{2}\right\rangle$ is a fuchsian group with fundamental polygon $D_{1} \cap D_{2}$.

\section{A THEORY OF NESTED GEODESICS IN $\mathbb{H}^{2}$}

Let $\left\{L_{i}\right\}$, for $i=1,2,3, \ldots$, be a sequence of geodesics in $\mathbb{H}^{2}$. We say that this sequence is a nested sequence of geodesics if $L_{i-1}$ and $L_{i+1}$ lie in different components of $H^{2}-L_{i}$ for each $i$, and if the $L_{i}$ are disjoint in $\overline{\mathbb{H}}^{2}$. 

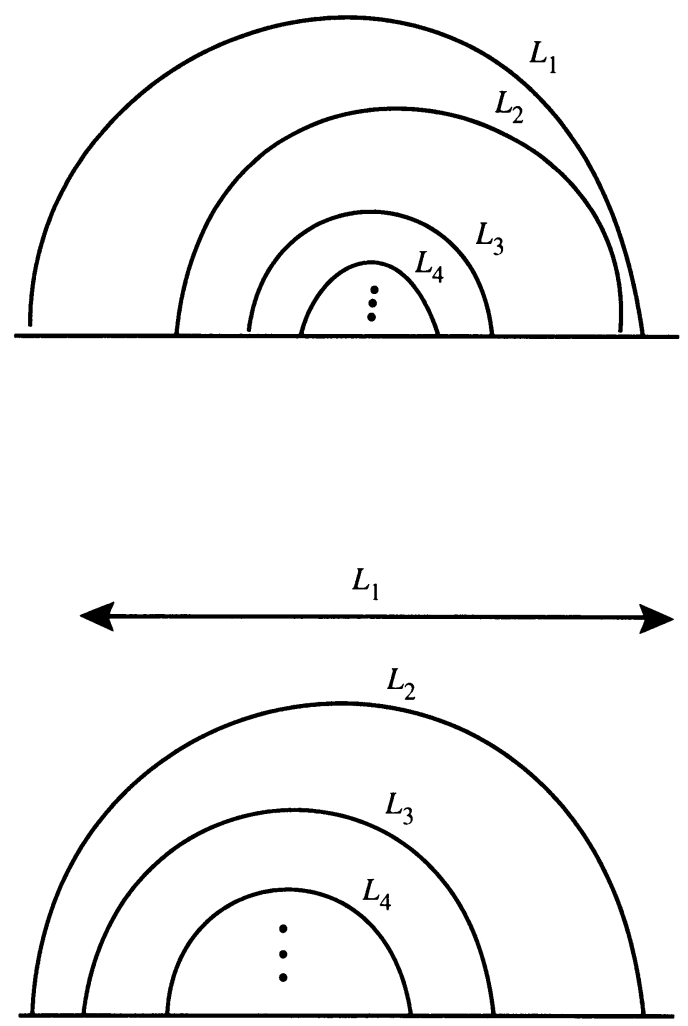

FIGURE 2

All of the theory in this section also works if we allow the first element $L_{1}$ of the sequence $\left\{L_{i}\right\}$ to be a horocycle. In this case the distance from $L_{1}$ to a geodesic disjoint from $L_{1}$ is, as usual, the length of the unique common orthogonal through $L_{1}$ and the other geodesic. We will need to use nested sequences with $L_{1}$ a horocycle when we consider tight flutes. We leave it to the reader to verify that the rest of the material in this section works if $L_{1}$ is a horocycle (Figure 2).

The nested sequence of geodesics $\left\{L_{i}\right\}$ converges to the geodesic $L$ if the endpoints of the $L_{i}$ converge to the endpoints of $L$ on the boundary of the hyperbolic plane, $\partial \mathbb{H}^{2}$. If the endpoints of the $L_{i}$ converge to a single point of the boundary $\partial \mathbb{H}^{2}$ then we say that the nested sequence of geodesics converges to a point (or converges to the boundary of the hyperbolic plane). Clearly, the limit of a nested sequence is unique and is one of the two above possibilities.

Proposition. Suppose $\left\{L_{i}\right\}$ is a nested sequence of geodesics that converges to the geodesic $L$. Then

$$
\lim _{i \rightarrow \infty} \rho\left(L_{i}, L\right)=0 .
$$

Proof. Observe that $\rho\left(L_{1}, L\right)>\rho\left(L_{2}, L\right)>\rho\left(L_{3}, L\right)>\cdots$. Thus the sequence $\left\{\rho\left(L_{i}, L\right)\right\}$ is a decreasing sequence of positive real numbers. Now, suppose this sequence is bounded from below, that is, $\rho\left(L_{i}, L\right) \rightarrow K>0$. This would mean there exists a tubular neighborhood of width $K$ about $L$ for which 


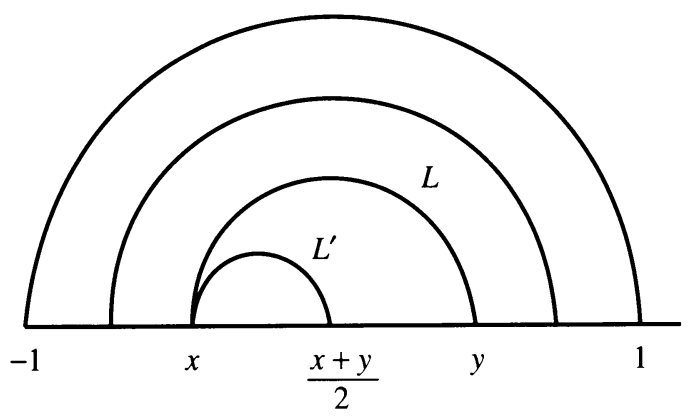

FIGURE 3

none of the $L_{i}$ can enter. Clearly this is impossible since the endpoints of the $L_{i}$ come arbitrarily close to the endpoints of $L$. Whence, the limit must be zero.

The converse to the above proposition is not true. Take any nested sequence of the geodesic $\left\{L_{i}\right\}=\left\{\left[x_{i}, y_{i}\right]\right\}$, where $-1<x_{i}<x_{i+1}<y_{i+1}<y_{i}<1$, which converges to a geodesic $L=[x, y]$. Construct the geodesic $L^{\prime}=\left[x, \frac{x+y}{2}\right]$. Since the distance $\rho\left(L^{\prime}, L\right)=0$, we conclude that the distances $\rho\left(L_{i}, L^{\prime}\right)$ go to zero. On the other hand, the $y_{i}$ do not converge to $\frac{x+y}{2}$, hence the nested sequence $\left\{L_{i}\right\}$ does not converge to $L^{\prime}$ (Figure 3 ).

Proposition. Let the sequence $\left\{L_{i}\right\}$ be a nested sequence of geodesics. Then the $L_{i}$ converge to a geodesic if and only if

$$
\lim _{i \rightarrow \infty} \rho\left(L_{1}, L_{i}\right)<\infty .
$$

Proof. Suppose the $L_{i} \rightarrow L$. Since the $L_{i}$ are nested we have

$$
\rho\left(L_{1}, L_{i}\right) \leq \rho\left(L_{1}, L\right) \text { for all } i=1,2,3, \ldots \text {. }
$$

Taking the limit in (3.1) as $i \rightarrow \infty$ and noting that the distances $\rho\left(L_{1}, L_{i}\right)$ increase with $i$, we conclude that the limit

$$
\lim _{i \rightarrow \infty} \rho\left(L_{1}, L_{i}\right)<\infty .
$$

For the converse, simply observe that if the $L_{i}$ converge to a boundary point then the distances $\rho\left(L_{1}, L_{i}\right)$ get arbitrarily large.

Let $\left\{L_{i}\right\}$ be a nested sequence of geodesics. We introduce an orientation (or direction) for each $L_{i}$ by designating $L_{i+1}$ to lie to the right of $L_{i}$. Let $\mathscr{O}_{i}$ be the unique common orthogonal between the geodesics $L_{i}$ and $L_{i+1}$. The distance from $\mathscr{O}_{i}$ to $\mathscr{O}_{i+1}$ is measured by traversing $L_{i+1}$, hence the following definition makes sense. Define

$$
s_{i}= \begin{cases}\rho\left(\mathscr{O}_{i}, \mathscr{O}_{i+1}\right) & \text { if } L_{i+1} \text { is traversed in the positive direction, } \\ -\rho\left(\mathscr{O}_{i}, \mathscr{O}_{i+1}\right) & \text { if } L_{i+1} \text { is traversed in the negative direction. }\end{cases}
$$

We call $s_{i}$ the slide (or twist) parameter for $L_{i+1}$. See Figure 4.

We would like to express nested sequences of geodesics in hyperbolic terms. The next proposition allows us to do exactly this. Set $d_{i}=\rho\left(L_{i}, L_{i+1}\right)$. 


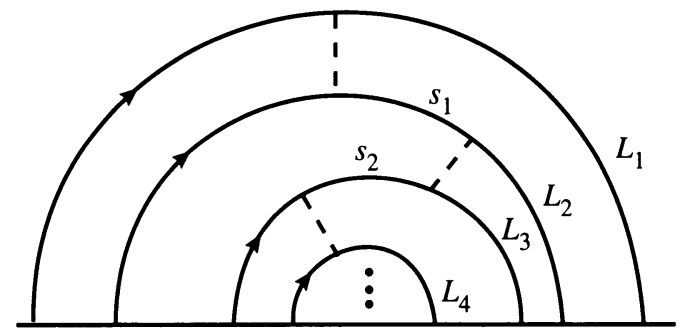

FIGURE 4

Proposition. The sequence of distances $\left\{d_{i}\right\}$ together with the sequence of slide parameters $\left\{s_{i}\right\}$, denoted $\left(\left(d_{i}\right),\left(s_{i}\right)\right.$ ), uniquely determine (up to an isometry of $\left.\mathbb{H}^{2}\right)$ a nested sequence of geodesics.

Proof. We work in the upper half-plane model. Suppose $\left\{L_{i}\right\}$ is a nested sequence of geodesics. Normalize by an element of $\operatorname{PSL}(2, \mathbb{R})$ so that $L_{1}=$ $[-1,1]$ oriented from -1 to 1 and the unique common orthogonal between $L_{1}$ and $L_{2}$ is $[0, \infty]$. The orientations induced on $\left\{L_{i}\right\}$ are as in Figure 4. Clearly $L_{2}$ is determined as soon as $d_{1}$ is specified. Next, $L_{3}$ is determined once we specify $s_{1}$ and $d_{2}$. In general, $L_{n}$ is determined once $\left\{d_{i}\right\}_{i=1}^{n-1}$ and $\left\{s_{i}\right\}_{i=1}^{n-2}$ are chosen.

We call the sequences $\left\{d_{i}\right\}$ and $\left\{s_{i}\right\}$ the coordinates of the nest $\left\{L_{i}\right\}$, and denote it by $\left(\left(d_{i}\right),\left(s_{i}\right)\right)$.

In the sequel, we will need to identify when a nested sequence of geodesics converges to a geodesic or to a point. The following propositions supply us with some tools.

Proposition. Suppose $\left\{L_{i}\right\}$ is a nested sequence of geodesics that converges to a geodesic. Then

$$
\sum_{1}^{\infty} \rho\left(L_{i}, L_{i+1}\right)<\infty
$$

Proof. First we claim that the following inequality holds,

$$
\sum_{1}^{m-1} \rho\left(L_{i}, L_{i+1}\right) \leq \rho\left(L_{1}, L_{m}\right) .
$$

Once we have verified (3.2) the proposition follows easily by letting $m$ go to infinity and noting that the limit

$$
\lim _{m \rightarrow \infty} \rho\left(L_{1}, L_{m}\right)
$$

exists since the $\left\{L_{i}\right\}$ converge to a geodesic.

To verify (3.2), we observe that the common orthogonal between $L_{1}$ and $L_{m}$ passes through each of the geodesics $L_{2}, L_{3}, \ldots, L_{m-1}$. Let $z_{i}$ be the point on $L_{i}$ that this common orthogonal intersects. Observe that the distance

$$
\rho\left(L_{1}, L_{m}\right)=\rho\left(z_{1}, z_{2}\right)+\rho\left(z_{2}, z_{3}\right)+\cdots+\rho\left(z_{m-1}, z_{m}\right) .
$$

On the other hand, the distances $\rho\left(z_{i}, z_{i+1}\right)$ must be at least as big as the length of the common orthogonal from $L_{i}$ to $L_{i+1}$, that is, $\rho\left(z_{i}, z_{i+1}\right) \geq \rho\left(L_{i}, L_{i+1}\right)$. This verifies (3.2). 
Proposition. Suppose $\left\{L_{i}\right\}$ is a nested sequence of geodesics such that $\sum d_{i}<\infty$ and $\sum\left|s_{i}\right|<\infty$. Then the nested sequence $\left\{L_{i}\right\}$ converges to a geodesic.

Proof. Let $\mathscr{O}_{i}$ be the common orthogonal to the geodesics $L_{i}$ and $L_{i+1}$ and set $z_{i}=\mathscr{O}_{i} \cap L_{i}$. Then we have

$$
\rho\left(L_{1}, L_{m}\right) \leq \rho\left(z_{1}, z_{m}\right),
$$

and hence since the geodesic joining $z_{1}$ and $z_{m}$ is shorter than any curve joining $z_{1}$ and $z_{m}$, we have

$$
\rho\left(L_{1}, L_{m}\right) \leq d_{1}+\left|s_{1}\right|+d_{2}+\left|s_{2}\right|+\cdots+d_{m-1}+\left|s_{m-1}\right|=\sum_{1}^{m-1} d_{i}+\sum_{1}^{m-1}\left|s_{i}\right| .
$$

Letting $m \rightarrow \infty$ in (3.3) we find that $\lim _{m \rightarrow \infty} \rho\left(L_{1}, L_{m}\right)<\infty$. Therefore the sequence $\left\{L_{i}\right\}$ converges to a geodesic.

We finish this section with some examples to illustrate that the converse statements to the previous propositions are not true.

Since the distance between two geodesics is a continuous function of the endpoints of the geodesics, we remark that given any two geodesics $M$ and $N$ we can construct a third geodesic $T$ which separates $M$ and $N$ such that the distances $\rho(M, T)$ and $\rho(T, N)$ are arbitrarily small. In fact, the absolute value of the slide parameter (the distance from the orthogonal of $M$ and $T$ to the common orthogonal of $T$ and $N$ ) can be made arbitrarily large. Using this fact we next construct an example to show that the series $\sum d_{i}<\infty$ is not sufficient to guarantee convergence of a nested sequence to a geodesic.

Consider a nested sequence of geodesics $\left\{L_{2 k-1}\right\}_{k=1}^{\infty}$ all having the same common orthogonal and converging to a point. For the pair $L_{2 k-1}$ and $L_{2 k+1}$ construct, as indicated above, a geodesic $L_{2 k}$ which comes within a distance of $1 /(2 k-1)^{2}$ to $L_{2 k-1}$ and a distance of $\frac{1}{(2 k)^{2}}$ to $L_{2 k+1}$. Do this for each $k$ and append all of these geodesics to form the new sequence $\left\{L_{i}\right\}$. Note that $\rho\left(L_{n}, L_{n+1}\right)<\frac{1}{n^{2}}$, and hence

$$
\sum \rho\left(L_{n}, L_{n+1}\right)<\infty .
$$

On the other hand, since the appended sequence $\left\{L_{i}\right\}$ has a subsequence that converges to a point, we conclude that the appended sequence converges to a point. Thus the series $\sum d_{i}$ converging is not sufficient to guarantee the convergence of the sequence $\left\{L_{i}\right\}$ to a geodesic.

Our next example illustrates the fact that a nested sequence can converge to a geodesic and yet the series $\sum\left|s_{i}\right|$ can diverge.

We start with a nested sequence of geodesics $\left\{L_{2 k-1}\right\}_{k=1}^{\infty}$ all having the same common orthogonal and converging to the geodesic $L$. Pair off the geodesics $L_{2 k-1}$ and $L_{2 k+1}$ as we did in the previous example. For each such pair construct the geodesic $L_{2 k}$ which separates $L_{2 k-1}$ from $L_{2 k+1}$ so that the distance from the common orthogonal of $L_{2 k-1}$ and $L_{2 k}$ to the common orthogonal of $L_{2 k}$ and $L_{2 k+1}$ is bounded from below by some positive constant which does not depend on the pair. Once again, append all of these geodesics to the sequence to get the new sequence $\left\{L_{i}\right\}$. We note that the appended sequence 
$\left\{L_{i}\right\}$ converges to $L$ and yet $\sum\left|s_{i}\right|=\infty$. Thus we have constructed an example with the desired properties.

\section{THE PROOF OF THE FUNDAMENTAL THEOREM (TheOREM 3)}

We remark that when the $G_{n}$ in Theorem 3 are pairs of pants, then $\mathbb{H}^{2} / G$ is a flute space. We will prove the fundamental theorem using a pair of lemmas.

Lemma. Suppose $G_{n}$, for $n=1$ and 2 , are fuchsian groups with

$$
N\left(G_{1}\right) \cap N\left(G_{2}\right)=A(g),
$$

where $\langle g\rangle$ is a primitive boundary hyperbolic subgroup in both $G_{1}$ and $G_{2}$. Suppose further that $I$ is an interval of discontinuity for $G_{2}$ which is not $G_{2}$ conjugate to the interval of discontinuity bounded by $A(g)$. Then $I$ is an interval of discontinuity for $\left\langle G_{1}, G_{2}\right\rangle$. In particular, if $\langle h\rangle$ is a primitive boundary hyperbolic subgroup in $G_{2}$ which is not $G_{2}$-conjugate to $\langle g\rangle$, then $\langle h\rangle$ is a primitive boundary hyperbolic subgroup in $\left\langle G_{1}, G_{2}\right\rangle$.

Proof. Let $H$ be the stabilizer of $I$ under $G_{2}$. We claim that $I$ is precisely invariant under $H$ in $\left\langle G_{1}, G_{2}\right\rangle$. Once we have proven this the lemma follows from elementary considerations. The rest of the proof will be devoted to proving the claim.

Let $g \in\left\langle G_{1}, G_{2}\right\rangle-H$. The only nontrivial possibility to check is when $g$ is a product of elements from both $G_{1}$ and $G_{2}$. First observe that an element of $G_{1}-\langle g\rangle$ takes $I$ into a $G_{1}$-interval of discontinuity which is bounded by a $G_{1}$-conjugate to $g$. Next observe that an element of $G_{2}$ will take this image of $I$ into a $G_{2}$-interval of discontinuity bounded by a $G_{2}$-conjugate of $g$. The above two observations used repeatedly will show that $g I \cap I=\varnothing$.

Main Lemma. Let $\left\{G_{n}\right\}_{n=1}^{\infty}$ be fuchsian groups with $\left\langle g_{n}\right\rangle$ and $\left\langle g_{n+1}\right\rangle$ nonconjugate primitive boundary hyperbolic subgroups in $G_{n}$ so that

$$
N\left(G_{n}\right) \cap N\left(G_{n+1}\right)=A\left(g_{n+1}\right) \text { for each } n .
$$

Let $G=\left\langle G_{n}\right\rangle_{n=1}^{\infty}$. Then for each $i$ the Nielsen region $N\left(G_{i}\right)$ is precisely invariant under the group $G_{i}$ in $G$.

Proof. Define $G^{n}$ to be $\left\langle G_{1}, G_{2}, \ldots, G_{n}\right\rangle$. If $g \in G_{i}$ then by definition $g N\left(G_{i}\right)=N\left(G_{i}\right)$. Next consider $g \in G-G_{i}$. Then $g \in G^{n}-G_{i}$ for large $n$ and thus it is enough to show that for all $i \leq n$ the Nielsen region $N\left(G_{i}\right)$ is precisely invariant under $G_{i}$ in $G^{n}$. The rest of our argument is devoted to proving this statement.

First, for $n=1$ the statement is obvious and for $n=2$ it follows from the combination theorem. We induct on $n$. Specifically, suppose that the Nielsen region $N\left(G_{i}\right)$ is precisely invariant under $G_{i}$ in $G^{n}$ for all $i$ satisfying $i \leq n$. We would like to show that $N\left(G_{i}\right)$ is precisely invariant under $G_{i}$ in $G^{n+1}$ for $i \leq n+1$.

We first observe that $G^{n+1}$ is by definition $\left\langle G^{n}, G_{n+1}\right\rangle$ and that

$$
N\left(G^{n}\right) \cap N\left(G_{n+1}\right)=A\left(g_{n+1}\right) .
$$

We note that the above expression follows from the fact that $g_{n+1}$ is a boundary hyperbolic (by the previous lemma) in $G^{n}$. Hence we can apply the KleinMaskit combination theorem to conclude that $G^{n+1}$ is a fuchsian group and 
that

$$
\begin{gathered}
N\left(G^{n}\right) \text { (respectively, } N\left(G_{n+1}\right) \text { ) is precisely invariant } \\
\text { under } \left.G^{n} \text { (respectively, } G_{n+1}\right) \text { in } G^{n+1} .
\end{gathered}
$$

Thus for $i=n+1$ the desired conclusion follows easily.

Next suppose $i \leq n$ and let $g \in G^{n+1}-G_{i}$. Note that

$$
G^{n+1}-G_{i}=\left(G^{n+1}-G^{n}\right) \cup\left(G^{n}-G_{i}\right) .
$$

Now if $g \in G^{n}-G_{i}$, then by the induction hypothesis $g N\left(G_{i}\right) \cap N\left(G_{i}\right)=\varnothing$. Otherwise $g \in G^{n+1}-G^{n}$ and, by (4.1), $g N\left(G^{n}\right) \cap N\left(G^{n}\right)=\varnothing$. Furthermore, since $G_{i}$ is a subgroup of $G^{n}$, we observe that $N\left(G_{i}\right) \subseteq N\left(G^{n}\right)$ and hence $g N\left(G_{i}\right) \cap N\left(G_{i}\right)=\varnothing$. This shows that $N\left(G_{i}\right)$ is precisely invariant under $G_{i}$ in $G^{n+1}$ for $i \leq n+1$. This finishes the induction step and the proof of the main lemma.

Proof of the fundamental Theorem 3. Let $x$ be a point in the interior of the Nielsen region $N\left(G_{1}\right)$. Since $G_{1}$ is a fuchsian group, there exists an open set $U \subset N\left(G_{1}\right)$ so that

$$
h U \cap U=\varnothing, \quad \text { for all but finitely many } h \in G_{1} .
$$

Furthermore, since the region $N\left(G_{1}\right)$ is precisely invariant under $G_{1}$ in $G$, we have that $g U \cap U=\varnothing$ for all but finitely many $g \in G$. Thus $G$ acts discontinuously at $x$, hence on all of $\mathbb{H}^{2}$. This shows that $G$ is a fuchsian group.

It is clear from the combination theorem that $\pi\left(A\left(g_{n}\right)\right)$ is a simple closed dividing geodesic.

Finally, since the Nielsen region $N\left(G_{i}\right)$ is precisely invariant under $G_{i}$ in $G$, we can conclude that $N(G) / G$ is topologically the union of the surfaces $\left\{N\left(G_{n}\right) / G_{n}\right\}$ and that $N\left(G_{n}\right) / G_{n}$ isometrically embeds in $N(G) / G$.

\section{THE PROOFS OF THEOREMS 1 AND 2}

We define the collar width of a nonelliptic (orientation preserving) isometry $\gamma$ of $\mathbb{H}^{2}$ to be

$$
c(\gamma)= \begin{cases}\log \operatorname{coth} T(\gamma) / 4 & \text { if } \gamma \text { is hyperbolic, } \\ \log 2 & \text { if } \gamma \text { is parabolic. }\end{cases}
$$

Recall that the axis of a hyperbolic element is denoted by $A(\gamma)$. If $\gamma$ is parabolic we define $A(\gamma)$ to be the boundary of the horodisc based at the fixed point of $\gamma$ whose projection to $\mathbb{H}^{2} /\langle\gamma\rangle$ has area one. The projection of $A(\gamma)$ to the quotient surface will be a simple closed curve of length one. If $\gamma$ is given by $z \mapsto z+1$ in the upper half-plane model, then $A(\gamma)$ will be the horizontal line of height one.

In order to prove Theorems 1 and 2, we need to be able to construct pairs of pants in a geometrically explicit way.

The Pair of Pants Theorem. Suppose $\gamma$ and $\beta$ are nonelliptic elements. Let $d$ be the hyperbolic distance between the axes of $\gamma$ and $\beta$. Then $(\gamma, \beta)$ form standard generators for a pair of pants if and only if $c(\gamma)+c(\beta) \leq d$, with equality if and only if the pair of pants is tight. 
Furthermore, $d$ projects to the distance between the boundary components which correspond to $\gamma$ and $\beta$, and the numbers $c(\gamma)$ and $c(\beta)$ determine the widths of disjoint collars on the quotient surface $\mathbb{H}^{2} /\langle\gamma, \beta\rangle$.

The above theorem for $\gamma$ and $\beta$ hyperbolic is proven in [B-2]. The proof for $\gamma$ or $\beta$ parabolic (along with other related results) will appear elsewhere.

An immediate consequence of this theorem is the following lemma.

Lemma. (I) Given any triple of positive real numbers $\left(c_{1}, c_{2}, d\right)$ satisfying $c_{1}+$ $c_{2} \leq d$, there exists a pair of pants (tight pants if and only if equality) having hyperbolic standard generators $(\gamma, \beta)$ so that $c(\gamma)=c_{1}, c(\beta)=c_{2}$, and $d$ is the distance from the axis of $\gamma$ to the axis of $\beta$.

(II) Given any pair of positive numbers $c$ and $d$ satisfying $c+\log 2 \leq d$, there exists a pair of pants (tight pants if and only if equality) having standard generators $(\gamma, \beta)$, where $\gamma$ is hyperbolic and $\beta$ is parabolic, so that $c(\gamma)=c$ and $d$ is the distance from $A(\beta)$ to the axis $A(\gamma)$.

For both (I) and (II), $d$ projects to the quotient to become the distance between the boundary components associated to $\gamma$ and $\beta$.

Proof of Theorem 1. In $\S 3$ we saw how to construct a nested sequence of geodesics $\left\{L_{i}\right\}$ with coordinates $\left(\left(d_{i}\right),\left(s_{i}\right)\right)$.

Now construct a pair of pants $G_{1}$ having standard generators $\left\{g_{1}, g_{2}\right\}$ satisfying the inequalities $c\left(g_{1}\right)<d_{1}$ and

$$
c\left(g_{2}\right)<\min \cdot\left\{d_{1}-c\left(g_{1}\right), d_{2}\right\},
$$

where the axis of $g_{1}$ is $L_{1}$ and the axis of $g_{2}$ is $L_{2}$. Clearly, we can do this since $c\left(g_{2}\right)<d_{1}-c\left(g_{1}\right)$. Notice that the axes of $g_{1}$ and $g_{2}$ are contained in the boundary of the Nielsen region for $G_{1}$.

Next, construct a hyperbolic element $g_{3}$ whose axis is $L_{3}$ having the property. that

$$
c\left(g_{3}\right)<\min \cdot\left\{d_{2}-c\left(g_{2}\right), d_{2}\right\} .
$$

Then $\left\{g_{2}, g_{3}\right\}$ are standard generators for a pair of pants $G_{2}$. Observe that the boundary of the Nielsen region of $G_{2}$ contains the axes of $g_{2}$ and $g_{3}$. Clearly we can continue this process indefinitely. We call the pair of pants at the $n$th stage of the construction $G_{n}$. We note that

$$
N\left(G_{n}\right) \cap N\left(G_{n+1}\right)=A\left(g_{n+1}\right) .
$$

Let $G=\left\langle G_{n}\right\rangle$ and let $P_{n}$ be the associated quotient surface for $G_{n}$.

Note that $G$ satisfies all of the hypotheses of the fundamental Theorem 3. Thus $G$ is a flute space and it is easy to see by our construction that the set $\left\{g_{n}\right\}$ are standard generators for $G$ relative to the pants decomposition $\left\{P_{n}\right\}$ having the desired coordinates $\left(\left(d_{i}\right),\left(s_{i}\right)\right)$.

Next suppose that $S$ is a tight flute with standard generators $\left\{g_{n}\right\}$ (see Figure 1). We note that the distances $\left\{d_{n}\right\}$ between the axes $A\left(g_{n}\right)\left(A\left(g_{1}\right)\right.$ is a horocycle) completely determine the translation lengths $T\left(g_{n}\right)$ for all $n \geq 2$. To see this, recall that a tight flute has a pants decomposition into tight pairs of pants. Now, by the pair of pants theorem, each tight pair of pants has standard generators $\left\{g_{n}, g_{n+1}\right\}$ satisfying $c\left(g_{n}\right)+c\left(g_{n+1}\right)=d_{n}$ for each $n$. In particular, $c\left(g_{1}\right)=\log 2$ and $d_{1}$ determine $c\left(g_{2}\right)$. Next $c\left(g_{2}\right)$ and $d_{2}$ determine $c\left(g_{3}\right)$, and so on. Thus all the collar widths are determined. Since $c\left(g_{n}\right)$ and $T\left(g_{n}\right)$ 
are monotonic functions of each other, we conclude that the translation lengths of the $g_{n}$ are determined once the $d_{n}$ are specified.

The following lemma tells us precisely what the collar widths $\left\{c\left(g_{n}\right)\right\}$ are in terms of the coordinates of the standard generators $\left\{g_{n}\right\}$ for $S$. We set $t_{n}=\sum_{i=1}^{n}(-1)^{i+1} d_{i}$.

Lemma. Let $S$ be a tight flute having standard generators $\left\{g_{n}\right\}$ with coordinates $\left(\left(d_{i}\right),\left(s_{i}\right)\right)$. Then

$$
c\left(g_{n}\right)=(-1)^{n}\left(t_{n-1}-\log 2\right) \text { for } n=2,3,4, \ldots .
$$

Proof of Lemma. Since $S$ is a tight flute, the pairs $\left(g_{n}, g_{n+1}\right)$ for each $n$ are standard generators for a tight pair of pants. Therefore the pair of pants theorem supplies us with the formula $c\left(g_{n+1}\right)=d_{n}-c\left(g_{n}\right)$. Hence, using this equation repeatedly, we have

$$
\begin{array}{rlr}
c\left(g_{n+1}\right) & =d_{n}-c\left(g_{n}\right)=d_{n}-\left(d_{n-1}-c\left(g_{n-1}\right)\right) \\
& =d_{n}-d_{n-1}+\left(d_{n-2}-c\left(g_{n-2}\right)\right) & \\
& =\cdots= \begin{cases}d_{n}-d_{n-1}+d_{n-2}-\cdots-d_{1}+c\left(g_{1}\right) & \text { for } n=2 k, \\
d_{n}-d_{n-1}+\cdots+d_{1}-c\left(g_{1}\right) & \text { for } n=2 k-1,\end{cases} \\
& = \begin{cases}-\left(t_{2 k}-c\left(g_{1}\right)\right) & \text { for } n=2 k, \\
\left(t_{2 k-1}-c\left(g_{1}\right)\right) & \text { for } n=2 k-1 .\end{cases}
\end{array}
$$

Finally, since $g_{1}$ is parabolic, $c\left(g_{1}\right)=\log 2$.

We are now prepared to prove Theorem 2.

Proof of Theorem 2. First we show that $(*)$ is necessary. The previous lemma, coupled with the fact that the collar width of an element is a positive quantity, give us

$$
t_{n-1}-\log 2=c\left(g_{n}\right)>0 \text { for } n \text { even. }
$$

Hence, $t_{n-1}>\log 2$ for $n$ even or equivalently $t_{n}>\log 2$ for $n$ odd. Similarly,

$$
-\left(t_{n-1}-\log 2\right)=c\left(g_{n}\right)>0 \text { for } n \text { odd. }
$$

Hence, $\log 2>t_{n-1}$ for $n$ odd or, in other words, $\log 2>t_{n}$ for $n$ even. This concludes the proof of the necessity of $(*)$.

For the sufficiency, assume that the $\left\{d_{i}\right\}$ are positive real numbers satisfying $(*)$. First construct the nested sequence of geodesics $\left\{L_{n}\right\}$, where $L_{1}$ is a horocycle, with coordinates $\left(\left(d_{i}\right),\left(s_{i}\right)\right)$. For each $L_{n}$, construct the hyperbolic element $g_{n}$ with axis $L_{n}$ and collar width

$$
c\left(g_{n}\right)=(-1)^{n}\left(t_{n-1}-\log 2\right) \text {. }
$$

Let $G_{n}$ be the group generated by $g_{n}$ and $g_{n+1}$. Note that condition $(*)$ guarantees that each of the collar widths make sense, that is, they are positive. We would like to show that $G_{n}$ is a hyperbolic structure for a tight pair of pants. To this end, note that

$$
\begin{aligned}
c\left(g_{n}\right)+c\left(g_{n+1}\right)= & (-1)^{n}\left(\sum_{i=1}^{n-1}(-1)^{i+1} d_{i}-\log 2\right) \\
& +(-1)^{n+1}\left(\sum_{i=1}^{n}(-1)^{i+1} d_{i}-\log 2\right)=d_{n} .
\end{aligned}
$$


Hence, by the pair of pants theorem, $\left(g_{n}, g_{n+1}\right)$ are standard generators for a pair of tight pants and thus $G_{n}$ is a hyperbolic structure for a pair of tight pants.

We set $G=\left\langle G_{n}\right\rangle$. From the fundamental Theorem $3, G$ is a hyperbolic structure for a flute space. Furthermore, by our construction, the set $\left\{g_{n}\right\}$ is a set of standard generators with coordinates $\left(\left(d_{i}\right),\left(s_{i}\right)\right)$. Finally, since $\mathbb{H}^{2} / G$ has pants decomposition $\left\{P_{i}\right\}$, where $P_{i}$ is a tight pair of pants $\left(P_{1}\right.$ is of type $(0 ; 2 ; 1))$, we conclude that $\mathbb{H}^{2} / G$ is a tight flute.

\section{THE PROOF OF THEOREM 4}

In order to prove Theorem 4 we need to better understand intervals of discontinuity for a flute space. The following lemmas achieve exactly this. Recall that $G^{n}$ is by definition the group $\left\langle G_{1}, G_{2}, \ldots, G_{n}\right\rangle$.

Lemma 1. An interval of discontinuity for a flute space $G=\left\langle G_{n}\right\rangle$ with standard generators $\left\{g_{n}\right\}$ is either the interval of a boundary hyperbolic or the interval bounded by a nest of axes $\left\{A\left(g_{n}^{\prime}\right)\right\}$, where $\left\langle g_{n+1}^{\prime}\right\rangle$ is $G^{n}$-conjugate to $\left\langle g_{n+1}\right\rangle$.

Proof. The main point here is that the only boundary hyperbolics of $G^{n}$ which are not boundary hyperbolics in $G$ are elements that are $G^{n}$-conjugate to $\left\langle g_{n+1}\right\rangle$.

Now suppose $I$ is an interval of discontinuity for $G$ which is not the interval for a boundary hyperbolic in $G$. Since $G^{n} \leq G$ and $G^{n} \rightarrow G$, we have that there exist intervals $\left\{I_{n}\right\}$, where $\bigcap I_{n}=I, I_{n} \supset I_{n+1}$ for each $n$, and $I_{n}$ is an interval of discontinuity for $G^{n}$. Now, $I_{n}$ is bounded by the axis of an element $g_{n+1}^{\prime}$, where $\left\langle g_{n+1}^{\prime}\right\rangle$ is $G^{n}$-conjugate to $\left\langle g_{n+1}\right\rangle$. Thus we have a sequence of axes $\left\{A\left(g_{n}^{\prime}\right)\right\}$. Furthermore, since $I_{n} \supset I_{n+1}$ and $\bigcap I_{n}=I$, this is a nested sequence which converges to the geodesic that bounds $I$.

Fix $\left\{g_{n}\right\}$ to be a set of standard generators for a flute space $G$. Lemma 1 makes it clear that nests of axes conjugate (by not necessarily the same element) to the axes of the standard generators will play an important role in our study of flute spaces. We let $\mathscr{L}=\mathscr{L}\left(\left\{g_{i}\right\}\right)$ be the set

$\left\{\left\{A\left(g_{i}^{\prime}\right)\right\}: g_{i}^{\prime}\right.$ is $G$-conjugate to $g_{i}$ for each $i$ and $\left\{A\left(g_{i}^{\prime}\right)\right\}$ is a nested sequence $\}$.

In other words, $\mathscr{L}$ is the set of all nested sequences of axes that are $G$-conjugate to the axes of the standard generators. Notice that the element that conjugates $\left\langle g_{i}\right\rangle$ to $\left\langle g_{i}^{\prime}\right\rangle$ need not be the same element that conjugates $\left\langle g_{i+1}\right\rangle$ to $\left\langle g_{i+1}^{\prime}\right\rangle$.

Lemma 2. Suppose $\left\{g_{n}\right\}$ is a set of standard generators for a flute space $G$. Furthermore, suppose that the nest of axes $\left\{A\left(g_{n}^{\prime}\right)\right\}$ converges to a geodesic $L$. Then the interval I bounded by $L$ is precisely invariant under the identity in $G$. In particular, this interval will be an interval of discontinuity for the nest.

Proof. Let $g$ be a nontrivial element of $G$. Then for a large enough $n, g$ is contained in $G^{n}-\left\langle g_{n+1}\right\rangle$. Hence, since $\left\langle g_{n+1}\right\rangle$ is $G^{n}$-conjugate to $\left\langle g_{n+1}^{\prime}\right\rangle$ and $g_{n+1}^{\prime}$ is a boundary hyperbolic for $G^{n}$, we have that the interval of discontinuity bounded by $A\left(g_{n+1}^{\prime}\right)$ is precisely invariant under $\left\langle g_{n+1}^{\prime}\right\rangle$ in $G^{n}$. Since $I$ is contained in the interval bounded by the axis $A\left(g_{n+1}^{\prime}\right)$, we can conclude that $g I \cap I=\varnothing$. 
Lemma 1 tells us that intervals of discontinuity for a flute space are either bounded by boundary hyperbolics or by nested sequences in $\mathscr{L}$. We are now ready to prove Theorem 4 .

Proof of Theorem 4. Since $G$ is a tight flute, it has no intervals of discontinuity coming from boundary hyperbolic elements. Thus by Lemma 1 the only intervals of discontinuity come from nests of axes in $\mathscr{L}$. On the other hand, observe that $d_{i}=\rho\left(g_{i}, g_{i+1}\right) \leq \rho\left(g_{i}^{\prime}, g_{i+1}^{\prime}\right)$. The inequality follows from the fact that the distance $d_{i}$ descends to the quotient surface to be the distance between the closed geodesics associated to $g_{i}$ and $g_{i+1}$. Hence the series $\sum d_{i}$ diverging implies that any nest in $\mathscr{L}$ goes to the boundary $\partial \mathbb{H}^{2}$. We conclude that $G$ is of the first kind.

If the two series $\sum d_{i}$ and $\sum\left|s_{i}\right|$ converge then the nest $\left\{A\left(g_{i}\right)\right\}$ converges to a geodesic and the interval on the boundary of $\mathbb{H}^{2}$ bounded by this geodesic is, by Lemma 2, an interval of discontinuity for $G$. Whence $G$ is of the second kind.

\section{THE PROOF OF THEOREM 5}

Suppose that $G$ and $\left\{D_{i}\right\}$ satisfy the hypotheses of Theorem 5 . We set $D^{n}=D_{1} \cap D_{2} \cap \cdots \cap D_{n}$. Note that after applying the combination theorem to $G_{1}$ and $G_{2}$ we have $D^{2} \cap B\left(g_{2}\right)=D^{1} \cap B\left(g_{2}\right)$, and in general after applying the combination theorem to the groups $G^{n-1}$ and $G_{n}$ we have $D^{n} \cap B\left(g_{n}\right)=$ $D^{n-1} \cap B\left(g_{n}\right)$, that is, $D^{n-1}$ and $D^{n}$ are the same in the half-space $B\left(g_{n}\right)$.

Proof of Theorem 5. Recall that $G^{n}$ is defined to be $\left\langle G_{1}, \ldots, G_{n}\right\rangle$. Observe that the sides of $D$ are paired by elements of $G$ and that $D$ is a convex hyperbolic polygon. Now, let $g$ be a nontrivial element of $G$. Then $g$ is contained in $G^{n}$ for a sufficiently large $n$. Hence $g D^{n} \cap D^{n}=\varnothing$ and therefore, since $D \subset D^{n}$, we have that $g D \cap D=\varnothing$.

In order to finish the proof of Theorem 5, we need a lemma which (roughly speaking) says that all nested sequences in $G$ go to the boundary of $\mathbb{H}^{2}$. We let $\mathscr{R}\left(G^{n}\right)$ be the set of all boundary half-spaces of $G^{n}$ with the boundary half-space of $g_{n+1}$ and its $G^{n}$-conjugates deleted. Let $W^{n}:=N\left(G^{n}\right) \cup \mathscr{R}\left(G^{n}\right)$.

Lemma (Properties of $\left.W^{n}\right)$. Suppose that $G$ satisfies the hypotheses of Theorem 5. Then

(i) $W^{n}$ is precisely invariant under $G^{n}$ in $G$;

(ii) $W^{1} \subset W^{2} \subset W^{3} \subset \cdots \subset W^{n} \subset \cdots$;

(iii) $D \cap W^{n}=D \cap B\left(g_{n+1}\right)$;

(iv) $W^{n} \rightarrow \mathbb{H}^{2}$ as $n \rightarrow \infty$. In fact, given a positive number $r$ there exists a positive integer $n$ so that $B_{r} \subset W^{n}$, where $B_{r}$ is the disc of radius $r$ centered at the origin of the hyperbolic plane.

Proof. Statement (i) follows from applying the Klein-Maskit combination theorem to the pair $G^{n}$ and $\left\langle G_{n+1}, G_{n+2}, \ldots\right\rangle$ (note that this later group is fuchsian by Theorem 3 ). The main observation being that the intersection of their Nielsen regions is the axis $A\left(g_{n+1}\right)$.

Next we prove statement (ii). Clearly the Nielsen region $N\left(G^{n}\right) \subset N\left(G^{n+1}\right)$, since $G^{n}$ is a subgroup of $G^{n+1}$. Also $\mathscr{R}\left(G^{n}\right) \subset \mathscr{R}\left(G^{n+1}\right)$, since an interval 
of discontinuity for $G^{n}$ not conjugate to $\left\langle g_{n+1}\right\rangle$ remains an interval of discontinuity for $G^{n+1}$ (see the lemma of $\S 4$ ). These two containments imply that $W^{n} \subset W^{n+1}$.

Property (iii) follows from the fact that $D$ does not intersect any of the $G^{n}$-conjugates of the boundary half-spaces of $g_{n+1}$ except $g_{n+1}$ itself.

Finally to prove (iv) assume that the sets $\left\{W^{n}\right\}$ do not exhaust all of $\mathbb{H}^{2}$; then since they form an increasing sequence of sets, there exists a nested sequence of axes $\left\{A\left(g_{n+1}^{\prime}\right)\right\}$ converging to a geodesic, where $g_{n+1}^{\prime}$ is $G^{n}$-conjugate to $g_{n+1}$. We conclude from this that the series $\sum \rho\left(g_{n}^{\prime}, g_{n+1}^{\prime}\right)<\infty$.

On the other hand, by assumption we know that

$$
\sum \rho\left(g_{n}^{\prime}, g_{n+1}^{\prime}\right) \geq \sum \rho\left(g_{n}, g_{n+1}\right)=\infty .
$$

The inequality is from hypothesis (II) in Theorem 5 . We have clearly reached a contradiction.

We return to the proof of Theorem 5. Let $x \in \mathbb{H}^{2}$. Statement (iv) in the above lemma tells us there exists a positive integer $n$ so that $x \in W^{n}$. Since $D^{n}$ is a fundamental polygon for $G^{n}$, there exists an element $g \in G^{n}$ so that $g(x) \in \overline{D^{n}}$. Furthermore, since $W^{n}$ is invariant under $G^{n}$ and $W^{n} \subset \bar{B}\left(g_{n+1}\right)$, we have $g(x) \in \overline{D^{n}} \cap \bar{B}\left(g_{n+1}\right)=\bar{D} \cap \bar{B}\left(g_{n+1}\right)$. Thus $g(x) \in \bar{D}$.

Finally we would like to show that the tesselation is locally finite. Let $K$ be a compact set in $\mathbb{H}^{2}$. Once again, by (iv) of the above lemma, there exists a positive integer $n$ so that $K \subset W^{n}$. The only $G$-translates of $D$ which are in $W^{n}$ are the $G^{n}$-translates of $D \cap W^{n}$. Next, observe that

$$
D \cap W^{n}=D \cap B\left(g_{n+1}\right)=D^{n} \cap B\left(g_{n+1}\right),
$$

where the first equality follows from (iii) of the lemma. Hence it is enough to show that $K$ meets only finitely many $G^{n}$-translates of $D^{n} \cap B\left(g_{n+1}\right)$. However this is clear since $D^{n}$ is a fundamental polygon for $G^{n}$ and thus its tesselation is locally finite. This concludes the proof of Theorem 5 .

\section{Corollaries}

Corollary to Theorem 2. If $\left\{d_{i}\right\}_{i=2}^{\infty}$ is a nonincreasing (or nondecreasing) sequence of positive numbers and $\left\{s_{i}\right\}$ is any sequence of real numbers, then there exists a positive real number $d_{1}$ so that $\left(\left(d_{i}\right),\left(s_{i}\right)\right)$ are coordinates for a standard set of generators on a tight flute.

Proof. Suppose the sequence $\left\{d_{i}\right\}_{i=2}^{\infty}$ is nonincreasing, that is, $d_{2} \geq d_{3} \geq \cdots \geq$ $d_{n} \geq \cdots$. If we can find a positive constant $d_{1}$ satisfying condition $(*)$ in Theorem 2 we will be done. Now either $d_{i} \rightarrow 0$ or $d_{i} \rightarrow K>0$.

If $d_{i} \rightarrow 0$, then the alternating series $\sum_{i=2}^{\infty}(-1)^{i+1} d_{i}$ converges. Hence set

$$
d_{1}=\log 2-\sum_{i=2}^{\infty}(-1)^{i+1} d_{i}, \quad \text { that is, } \sum_{i=1}^{\infty}(-1)^{i+1} d_{i}=\log 2 .
$$

Note that $\log 2$ is smaller than all the odd partial sums and bigger than all the even partial sums, that is, $\left\{d_{i}\right\}_{i=1}^{\infty}$ satisfies $(*)$.

If $d_{i} \rightarrow K>0$, then the alternating series $\sum_{i=2}^{\infty}(-1)^{i+1} d_{i}$ limits to two values. Then there will be an interval of $d_{1}$ 's to pick from so that

$$
t_{2 k}<t_{2 k+2}<\cdots<t_{2 k+3}<t_{2 k+1}<t_{2 k-1} \text { for all } k \text {, }
$$


where $t_{n}$ is the $n$th partial sum of the series $\sum_{i=1}^{\infty}(-1)^{i+1} d_{i}$. Hence, condition $(*)$ is satisfied again.

For a nondecreasing sequence $\left\{d_{i}\right\}_{i=2}^{\infty}$ of positive numbers, simply take any real number $d_{1}$ that satisfies $\log 2<d_{1}<\log 2+d_{2}$. Note that since the $d_{i}$ are nondecreasing the even partial sums of $\sum_{i=1}^{\infty}(-1)^{i+1} d_{i}$ are less than $\log 2$ and decreasing, and the odd partial sums are bigger than $\log 2$ and increasing. Thus $\left\{d_{i}\right\}_{i=1}^{\infty}$ once again satisfies $(*)$.

The following proposition relates the $\left(d_{i}\right)$ coordinates for standard generators $\left\{g_{i}\right\}_{i=1}^{\infty}$ on a tight flute with the translation lengths of these standard generators. Recall that $t_{n}=\sum_{i=1}^{n}(-1)^{i+1} d_{i}$. Of course, regardless of being a tight flute or not, if $d_{i} \rightarrow 0$ then the collar widths $c\left(g_{i}\right) \rightarrow 0$ and hence the translation lengths $T\left(g_{i}\right) \rightarrow \infty$.

Proposition. Suppose $\left\{g_{i}\right\}$ is a set of standard generators for a tight flute having coordinates $\left(\left(d_{i}\right),\left(s_{i}\right)\right)$.

(i) If $\left\{d_{i}\right\}_{i=1}^{\infty}$ is a nondecreasing sequence such that the distances $d_{i} \rightarrow \infty$, then the translation lengths $T\left(g_{i}\right) \rightarrow 0$.

(ii) If $\left\{d_{i}\right\}_{i=1}^{\infty}$ is a constant sequence, then $T\left(g_{i}\right)$ takes on at most two values, for all $i$. In particular, $\left\{T\left(g_{i}\right)\right\}$ is bounded from above and below.

Proof. Recall the formula $c\left(g_{n+1}\right)=\left|t_{n}-\log 2\right|$ derived in a lemma for $\S 5$.

For (i) observe that the even sums $t_{2 k} \rightarrow-\infty$ and the odd sums $t_{2 k-1} \rightarrow \infty$. Hence, we have $c\left(g_{i+1}\right) \rightarrow \infty$ and thus the translation lengths $T\left(g_{i}\right) \rightarrow 0$.

To prove (ii), set $d_{i}=d$. Then the even sums $t_{2 k}=0$ and the odd sums $t_{2 k-1}=d$. Hence, we have

$$
c\left(g_{i+1}\right)= \begin{cases}\log 2 & \text { for } i \text { even } \\ d-\log 2 & \text { for } i \text { odd }\end{cases}
$$

We conclude that the translation lengths $\left\{T\left(g_{i}\right)\right\}$ take on at most two values.

\section{EXAMPLES}

The first two examples of this section are meant to illuminate some of the theorems of this paper. The last example is an application of the theory developed.

An example related to Theorem 5. The following example will show that Theorem 5 does not hold if the series $\sum \rho\left(g_{n}, g_{n+1}\right)$ converges. First, we construct a fundamental polygon for a pair of pants. We remind the reader that boundary element means either a boundary hyperbolic or a parabolic element.

Example. Let $(\gamma, \beta)$ be standard generators for a pair of pants $\Gamma$ with axes and orientations depicted in Figure 5. Set $\eta=(\beta \gamma)^{-1}$. We say that $\Gamma$ is normalized and we work in the upper half-plane model (Figure 5). 


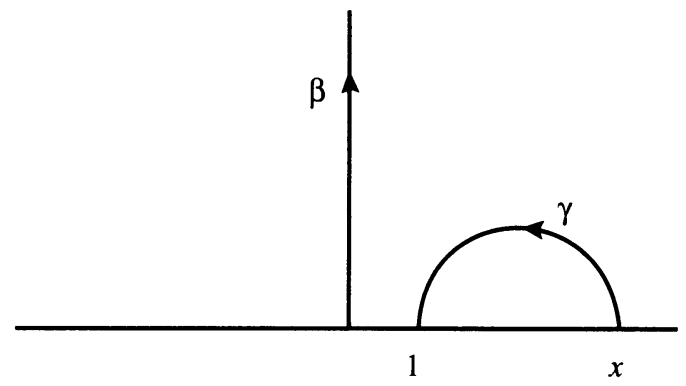

FIGURE 5

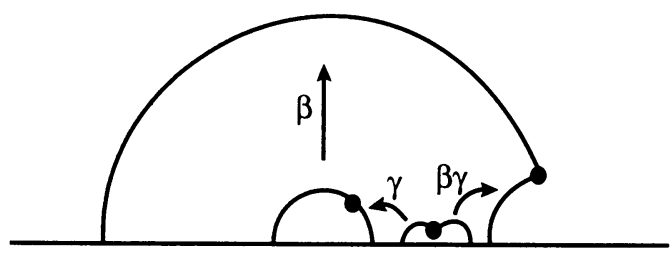

FIGURE 6

Let $\Sigma$ be the common orthogonal to the axes $A(\gamma)$ and $A(\beta)$. Consider the image of $\Sigma$ under $\beta, \beta(\Sigma)$. The right endpoint of $\beta(\Sigma)$ lies to the right of the right endpoint of $A(\eta)$. To see this, note that the group $\langle\gamma, \beta\rangle$ is an index two subgroup of the reflection group generated by the reflections in the common orthogonals to the axes of $\gamma, \beta$, and $\eta$. With this point of view, we can write $\beta$ as reflection in $\Sigma$ followed by reflection in the common orthogonal of the axes $A(\beta)$ and $A(\eta)$ (see the paper [B-2] for more details). Clearly the right endpoint of $\beta(\Sigma)$ lies to the right of the right endpoint of $A(\eta)$.

Next, we draw the common orthogonal to $A(\eta)$ and $\beta(\Sigma)$ and denote by $\beta(z)$ the point of intersection between this common orthogonal and $\beta(\Sigma)$. Hence $z$ is a point on $\Sigma$ that divides $\Sigma$ into two geodesic rays; call them $s_{2}$ and $s_{1}^{\prime}$ as in Figure 6 . Let $s_{3}$ be the common orthogonal segment between the axis $A(\eta)$ and the geodesic $\beta(\Sigma)$. Set $s_{2}^{\prime}=\beta\left(s_{2}\right)$ and $s_{1}=\gamma^{-1}\left(s_{1}^{\prime}\right)$. Finally, let $s_{3}^{\prime}$ be $\eta\left(s_{3}\right)$. Since $\eta \beta \gamma=1, \eta$ takes the vertex point $\beta(z)$ to the point $\gamma^{-1}(z)$. We claim that the angle made at the vertex $\gamma^{-1}(z)$ is $\pi / 2$. To see this, consider the (oriented) angle between the sides $\eta^{-1}\left(s_{1}\right)$ and $\eta^{-1}\left(s_{3}^{\prime}\right)$. Since $\eta^{-1}=\beta \gamma$ and $\eta^{-1}\left(s_{3}^{\prime}\right)=s_{3}$, this is the same as looking at the angle between $\beta \gamma\left(s_{1}\right)$ and $s_{3}$. But $\gamma\left(s_{1}\right)=s_{1}^{\prime}$ lies on $\Sigma$; hence $\beta \gamma\left(s_{1}\right)$ is the line segment on $\beta(\Sigma)$ which does not overlap $s_{2}^{\prime}$. We conclude that the angle between the sides $s_{1}$ and $s_{3}^{\prime}$ is the same as the angle between $\beta(\Sigma)$ and $s_{3}$, which is $\pi / 2$.

Now consider the polygon $P$ bounded by the sides constructed above having the identifications indicated in Figure 6. Observe that the hypotheses to Poincaré's theorem are all satisfied and thus $P$ is a fundamental polygon for the pair of pants.

If the group $\Gamma$ is not normalized, then we normalize (conjugate) it by an element $f$ of $\operatorname{PSL}(2, \mathbb{R})$ so that its standard generators are as in Figure 5. Next, we construct the fundamental polygon $P$ as above and translate it by $f^{-1}$. Since $f$ is an isometry of $\mathbb{H}^{2}$, this new polygon is a fundamental polygon for $\Gamma$. Once again we name this polygon $P$. We say that $P$ is the fundamental polygon associated to $(\gamma, \beta)$. 
Observe that two of the sides of $P$ lie on the common orthogonal geodesic to the axes of the standard generators $\gamma$ and $\beta$. Hence, in summary we have shown that given a standard set of generators $(\gamma, \beta)$ for a pair of pants, we can construct a fundamental polygon with two of its sides lying on the common orthogonal geodesic between the axes $A(\gamma)$ and $A(\beta)$.

Consider a flute space $G$ with standard generators $\left\{g_{n}\right\}$ having coordinates $d_{n}=1 / n^{2}$ and $s_{n}=0$. The important point to keep in mind is that the twist parameters have all been set equal to zero and hence all the axes of the $g_{n}$ have the same common orthogonal between them, call it $L$.

Construct for each pair of pants $G_{n}=\left\langle g_{n}, g_{n+1}\right\rangle$ the fundamental polygon $\left\{D_{n}\right\}$ associated to $\left(g_{n}, g_{n+1}\right)$. We note that the $\left\{D_{n}\right\}$ pairwise satisfy the compatibility conditions in the combination theorem. We set $D=\bigcap D_{n}$.

Note that the polygon $D$ has infinitely many sides which lie on the same geodesic $L$. Furthermore, since the nested sequence of axes $\left\{A\left(g_{i}\right)\right\}$ converges to a geodesic, the infinitely many sides which lie on $L$ must accumulate to a point of $\mathbb{H}^{2}$. We conclude that the action of $G$ on the polygon $D$ is not locally finite. Thus this conclusion of the combination theorem does not in general hold.

A tight flute of the second kind with a standard set of generators that converge to the boundary of the hyperbolic plane. Our next example illustrates the point that the nested sequence of axes for a standard set of generators on a tight flute may converge to the boundary of $\mathbb{H}^{2}$ even though $G$ is of the second kind. The crucial point here is that there are nested sequences of geodesics that converge to the boundary of $\mathbb{H}^{2}$ and yet the sum of the distances between the geodesics in the nest converge. Thus Theorem 4 is not valid if we replace the divergence of the series $\sum d_{i}$ with the condition that the axes $\left\{A\left(g_{i}\right)\right\}$ go to the boundary of $\mathbb{H}^{2}$.

Example. Let $G$ be a tight flute with standard generators $\left\{g_{n}\right\}$ having coordinates $d_{n}=1 / n^{2}$ for $n \geq 2$ and $s_{n}=0$. Note that by the proof of the corollary in $\S 8, d_{1}$ is determined. Orient the $g_{n}$ 's so that the axis $A\left(g_{n+1}\right)$ lies to the right of $A\left(g_{n}\right)$. Now, conjugate the generators $g_{2}, \ldots, g_{n}, \ldots$ by $g_{2}$. We get a new set of generators $\left\{g_{n}^{\prime}\right\}$ for $G$, namely

$$
g_{n}^{\prime}= \begin{cases}g_{n} & \text { if } n=1,2, \\ g_{2} g_{n} g_{2}^{-1} & \text { if } n \geq 3 .\end{cases}
$$

It is easy to check that the $\left\{g_{n}^{\prime}\right\}$ form standard generators for $G$ with coordinates $d_{n}^{\prime}=d_{n}$ and

$$
s_{n}^{\prime}= \begin{cases}s_{n}=0 & \text { if } n \neq 1 \\ T\left(g_{2}\right) & \text { if } n=1\end{cases}
$$

Next, conjugate the generators $\left\langle g_{4}^{\prime}, \ldots, g_{n}^{\prime}, \ldots\right\rangle$ by $g_{3}^{\prime}$. We get a new set of generators $\left\{g_{n}^{\prime \prime}\right\}$ given by

$$
g_{n}^{\prime \prime}= \begin{cases}g_{n}^{\prime} & \text { if } n=1,2,3, \\ g_{3}^{\prime} g_{n}^{\prime} g_{3}^{\prime-1} & \text { if } n \geq 4 .\end{cases}
$$

Once again note that the $\left\{g_{n}^{\prime \prime}\right\}$ form a standard set of generators for $G$ with 
coordinates $d_{n}^{\prime \prime}=d_{n}^{\prime}$ and

$$
s_{n}^{\prime \prime}= \begin{cases}s_{n}^{\prime} & \text { if } n \neq 2 \\ T\left(g_{3}\right) & \text { if } n=2 .\end{cases}
$$

If we continue this process ad infinitum, we get a new standard set of generators for $G$ with coordinates $\left\{d_{n}\right\}$ and $\left\{s_{n}\right\}=\left\{T\left(g_{n+1}\right)\right\}$. Since $d_{n} \rightarrow 0$, the translation lengths $T\left(g_{n+1}\right) \rightarrow \infty$ and hence $s_{n} \rightarrow \infty$.

Finally, we claim that the nest of axes for this standard set of generators goes to the boundary of $\mathbb{H}^{2}$. To see this, let $\Sigma_{n}$ be the common orthogonal geodesic from $A\left(g_{n}\right)$ to $A\left(g_{n+1}\right)$. Since the $s_{n}$ 's are all positive and since $s_{n} \rightarrow \infty$, the $\Sigma_{n}$ 's form a nested sequence of geodesics which go to the boundary of the hyperbolic plane. It follows that the nest of axes for this new set of standard generators goes to the boundary of the hyperbolic plane.

A type preserving isomorphism which is not a topological deformation. Let $\Phi$ : $G \rightarrow G^{\prime}$ be an isomorphism between two torsion-free fuchsian groups. Recall that $\Phi$ is said to be a type preserving isomorphism if boundary hyperbolics correspond to boundary hyperbolics and parabolics correspond to parabolics. We say that $\Phi$ is a topological deformation if there exists a homeomorphism $f: \overline{\mathbb{H}}^{2} \rightarrow \overline{\mathbb{H}}^{2}$ so that

$$
\Phi(g)(z)=f g f^{-1}(z) \text { for all } z \in \overline{\mathbb{H}}^{2} \text { and } g \in G .
$$

A central theorem in the theory of fuchsian groups and Teichmüller spaces is the Nielsen isomorphism theorem.

Nielsen isomorphism theorem. Suppose $\Phi: G \rightarrow G^{\prime}$ is a type preserving isomorphism between two finitely generated fuchsian groups. Then $\Phi$ is a topological deformation.

We will construct a type preserving isomorphism $\Phi$ between two torsion-free fuchsian groups which is not a topological deformation, thus showing that the Nielsen isomorphism theorem does not hold for infinitely generated fuchsian groups.

Example. Let $G$ be the tight flute with standard generators $\left\{g_{n}\right\}$ having coordinates $d_{n}=1 / n^{2}$ for $n \geq 2$ and $s_{n}=0$ (recall from the proof of the corollary in $\S 8$ that $d_{1}$ is determined if $d_{n}$ monotonically decreases to zero). Theorem 4 tells us that $G$ is of the second kind.

Next, let $G^{\prime}$ be a tight flute with standard generators $\left\{g_{n}^{\prime}\right\}$ having coordinates $d_{n}^{\prime}=1 / n$ and $s_{n}^{\prime}=0$. The group $G^{\prime}$ is of the first kind by Theorem 4 .

Since $\left(g_{n}, g_{n+1}\right)$ are standard generators we know that after possibly changing the orientations of $g_{n}$ and $g_{n+1}$, the product $g_{n} g_{n+1}$ is parabolic. Thus, without loss of information we can assume that $g_{n}$ and $g_{n+1}$ are oriented so that the product $g_{n} g_{n+1}$ is parabolic. We do the same thing for the standard generators $\left\{g_{n}^{\prime}\right\}$ of $G^{\prime}$.

It is clear by their construction that the groups $G$ and $G^{\prime}$ are isomorphic by the map $\Phi: G \rightarrow G^{\prime}$ which takes the generator $g_{n}$ to the generator $g_{n}^{\prime}$.

We would like to show that $\Phi$ is type preserving. By construction, we know that $G$ and $G^{\prime}$ have no boundary hyperbolics. Also $\Phi$ sends the parabolic $g_{1}$ to the parabolic $g_{1}^{\prime}$. Thus we need only show that $\Phi$ preserves the rest of the parabolics. 
First we note that the elements $g_{n} g_{n+1}$ and their inverses $g_{n+1}^{-1} g_{n}^{-1}$ represent all the conjugacy classes of parabolic elements in $G$; that is, any parabolic element $g \in G$ is equal to $h g_{n} g_{n+1} h^{-1}$ or $h g_{n+1}^{-1} g_{n}^{-1} h^{-1}$ for some $h \in G$. Of course the same statement holds for $G^{\prime}$. Since any isomorphism preserves the type of a conjugacy class, it is enough to show that $\Phi$ takes the parabolic element $g_{n} g_{n+1}$ to a parabolic element of $G^{\prime}$ (note: we need not consider the inverses $g_{n+1}^{-1} g_{n}^{-1}$ because

$$
\boldsymbol{\Phi}\left(g_{n+1}^{-1} g_{n}^{-1}\right)=\boldsymbol{\Phi}\left(\left(g_{n} g_{n+1}\right)^{-1}\right)=\left(\Phi\left(g_{n} g_{n+1}\right)\right)^{-1},
$$

and the inverse of a parabolic element is parabolic).

Now,

$$
\Phi\left(g_{n} g_{n+1}\right)=\Phi\left(g_{n}\right) \Phi\left(g_{n+1}\right)=g_{n}^{\prime} g_{n+1}^{\prime} ;
$$

but $\left(g_{n}^{\prime}, g_{n+1}^{\prime}\right)$ are standard generators for $G_{n}^{\prime}$. Hence, $g_{n}^{\prime} g_{n+1}^{\prime}$ is a parabolic element of $G$. We conclude that $\Phi$ is a type preserving isomorphism.

On the other hand, $\Phi$ is not a topological deformation. To see this, suppose there exists a homeomorphism $f: \overline{\mathbb{H}}^{2} \rightarrow \overline{\mathbb{H}}^{2}$ so that $\Phi(g)(z)=f g f^{-1}(z)$ for all $z \in \overline{\mathbb{H}}^{2}$ and $g \in G ; f$ would have to take the set of discontinuity of $G$ to the set of discontinuity of $G^{\prime}$. This is clearly impossible since $G$ is of the second kind and $G^{\prime}$ is of the first kind.

\section{REFERENCES}

[A] W. Abikoff, The real analytic theory of Teichmüller space, Lecture Notes in Math., vol. 820, Springer-Verlag, New York, 1980.

[A-S] L. Ahlfors and L. Sario, Riemann surfaces, Princeton Univ. Press, Princeton, N.J., 1960.

[B-1] A. Basmajian, Hyperbolic invariants for infinitely generated Fuchsian groups, Ph.D. thesis, Stony Brook University, 1987.

[B-2] _ Constructing pairs of pants, Ann. Acad. Sci. Fenn. Ser. A I Math. 15 (1990), 65-74.

[Be] A. Beardon, The geometry of discrete groups, Springer-Verlag, New York, 1983.

[G] F. Gardiner, Teichmüller theory and quadratic differentials, Wiley, New York, 1987.

[K] L. Keen, On infinitely generated Fuchsian groups, J. Indian Math. Soc. 35 (1971), 67-85.

[M] B. Maskit, Kleinian groups, Springer-Verlag, New York, 1987.

[N-S] M. Nakai and L. Sario, Classification theory of Riemann surfaces, Springer-Verlag, New York, 1970.

[P-1] N. Purzitsky, A cutting and pasting of non-compact polygons with applications to Fuchsian groups, Acta Math. 143 (1979), 233-250.

[P-2] _ Fricke polygons for infinitely generated fuchsian groups. I, preprint.

[T] W. Thurston, The geometry and topology of 3-manifolds, Lecture Notes, 1977.

Department of Mathematics, University of Oklahoma, Norman, Oklahoma 73019

E-mail address: abasmajian@nsfuvax.math.uoknor.edu 